\title{
Weak Poincaré Inequalities in the Absence of Spectral Gaps
}

\author{
Jonathan Ben-Artzi® and Amit Einav
}

\begin{abstract}
For generators of Markov semigroups which lack a spectral gap, it is shown how bounds on the density of states near zero lead to a socalled weak Poincaré inequality (WPI), originally introduced by Liggett (Ann Probab 19(3):935-959, 1991). Applications to general classes of constant coefficient pseudodifferential operators are studied. Particular examples are the heat semigroup and the semigroup generated by the fractional Laplacian in the whole space, where the optimal decay rates are recovered. Moreover, the classical Nash inequality appears as a special case of the WPI for the heat semigroup.
\end{abstract}

Mathematics Subject Classification. 39B62, 37A30, 35J05, 47D07.

\section{Contents}

1. Introduction and Statement of Results 359

2. Poincaré Inequalities 364

3. Proofs of the Theorems 366

4. Examples 370

Acknowledgements $\quad 373$

$\begin{array}{ll}\text { References } & 374\end{array}$

\section{Introduction and Statement of Results}

In this note, we study how the well-known equivalence between spectral gaps, Poincaré inequalities and exponential rates of decay to equilibrium extends to systems which lack a spectral gap but have a bounded density of states near 0 . Our main result relies solely on our ability to "differentiate" the resolution of 
the identity of a given operator. It is thus quite general and covers important examples such as Markov semigroups.

Our setup is as follows: let $M$ be a manifold with Borel measure $\mathrm{d} \mu$, $\mathcal{H}=L^{2}(M, \mathrm{~d} \mu ; \mathbb{R})$ equipped with scalar product $(\cdot, \cdot)_{\mathcal{H}}$. We assume that $H: D(H) \subset \mathcal{H} \rightarrow \mathcal{H}$ is a self-adjoint, nonnegative operator, so that $-H$ is the infinitesimal generator of a Markov semigroup $\left(P_{t}\right)_{t \geq 0}$, whose invariant measure is $\mathrm{d} \mu$, i.e., for every $u$ that is bounded and nonnegative $\int_{M} P_{t} u \mathrm{~d} \mu=\int_{M} u \mathrm{~d} \mu$ for any $t \geq 0$. Let $\{E(\lambda)\}_{\lambda \geq 0}$ be the resolution of the identity of $H$, and let the associated Dirichlet form be

$$
\mathcal{E}(u):=\int_{M}\left(H^{1 / 2} u\right)^{2} \mathrm{~d} \mu .
$$

As stated above, instead of assuming a spectral gap, we assume the opposite: $H$ has continuous spectrum in a neighborhood of 0 . (And 0 itself is possibly an eigenvalue.) We show that an appropriate estimate of the density of the spectrum near 0 leads to a weaker version of the Poincaré inequality (also known as a weak Poincaré inequality, defined in Definition 1.3). This, in turn, leads to an algebraic decay rate for the associated semigroup.

In this paper, we employ the following definition for the variance of a given function $u \in \mathcal{H}$ :

$$
\operatorname{Var}(u):=\int_{M}(u-E(\{0\}) u)^{2} \mathrm{~d} \mu
$$

where $E(\{0\})$ is the projection onto the kernel of $H$. In the case where the kernel only consists of constant functions and $\mu$ is a probability measure, this definition coincides with the standard definition, see [3, §4.2.1]. We discuss the significance of the resolution of the identity of $H$ (and in particular the projection onto its kernel) and its relationship with functional inequalities and decay rates in Sect. 2.3.

We can now recall the classical Poincaré inequality (again, see $[3, \S 4.2 .1]$ ):

Definition 1.1 (Poincaré Inequality). We say that $H$ satisfies a Poincaré inequality if there exists $C>0$ such that

$$
\operatorname{Var}(u) \leq C \mathcal{E}(u), \quad \forall u \in D(\mathcal{E}),
$$

where $C$ does not depend on $u$.

Remark 1.2. The topology of $D(\mathcal{E})$ is the graph norm topology generated by $\|\cdot\|_{\mathcal{H}}^{2}+\mathcal{E}(\cdot)$, see $[3, \S 3.1 .4]$.

The definition of a "weak Poincaré inequality" is somewhat ambiguous. This is addressed in further detail in Sect. 2.3. We adopt the following definition, motivated by Liggett [13, Equation (2.3)]:

Definition 1.3 (Weak Poincaré Inequality). Let $\Phi: \mathcal{H} \rightarrow[0, \infty]$ satisfy $\Phi(u)<$ $\infty$ on a dense subset of $D(\mathcal{E})$. Let $p \in(1, \infty)$. We say that $H$ satisfies a $(\Phi, p)$-weak Poincaré inequality $((\Phi, p)$-WPI) if there exists $C>0$ such that

$$
\operatorname{Var}(u) \leq C \mathcal{E}(u)^{1 / p} \Phi(u)^{1 / q}, \quad \forall u \in D(\mathcal{E}),
$$

where $C$ does not depend on $u$ and where $1 / p+1 / q=1$. 
Remark 1.4. Note that (1.1) is meaningful only on a dense subset of $D(\mathcal{E})$ where $\Phi<+\infty$.

\subsection{The Hilbertian Case}

We start our discussion by considering the purely Hilbertian case, i.e., we consider generators with density of states that are defined on subspaces which respect the Hilbert structure of $\mathcal{H}$, such as Sobolev spaces or weighted spaces. Our basic assumption is:

Assumption A1. There exists a dense subspace $\mathcal{X} \subset \mathcal{H}$ such that

(1) $\mathcal{X} \cap D(\mathcal{E})$ is dense in $D(\mathcal{E})$ (in the topology of $D(\mathcal{E})$ ),

(2) for some constants $r>0, C_{1}>0$ and $\alpha>-1$,

the mapping $\lambda \mapsto \frac{\mathrm{d}}{\mathrm{d} \lambda}(E(\lambda) u, v)_{\mathcal{H}}$ is continuous on $(0, r)$ for every $u, v \in \mathcal{X}$ and satisfies

$$
\left|\frac{\mathrm{d}}{\mathrm{d} \lambda}(E(\lambda) u, v)_{\mathcal{H}}\right| \leq C_{1} \lambda^{\alpha}\|u\|_{\mathcal{X}}\|v\|_{\mathcal{X}}, \quad \forall u, v \in \mathcal{X}, \forall \lambda \in(0, r) .
$$

Remark 1.5. We refer to the bilinear form $\frac{\mathrm{d}}{\mathrm{d} \lambda}(E(\lambda) \cdot, \cdot)_{\mathcal{H}}$ as the density of states (DoS) of $H$ at $\lambda$. Note that if the DoS satisfies a bound as in (1.2) and $\mathcal{X}$ has a norm compatible with (and stronger than) the norm on $\mathcal{H}$, then it induces an operator $\mathcal{X} \rightarrow \mathcal{X}^{*}$ by the Riesz representation theorem.

We can finally state our main results on how (1.2) leads to a $(\Phi, p)$ WPI (Theorem 1.6) and, in turn, an explicit rate of decay (Theorem 1.7). Theorem 1.6 will be further generalized in Theorem 1.9 and then again in Proposition 1.13 where a precise constant in the WPI is obtained. The decay rates presented in Theorem 1.7 apply to the Markov semigroup generated by $H$.

Theorem 1.6. If Assumption $\mathrm{A} 1$ holds, then $H$ satisfies a $(\Phi, p)$-weak Poincaré inequality with $\Phi(u)=\|u\|_{\mathcal{X}}^{2}($ and $\Phi(u)=+\infty$ if $u \in \mathcal{H} \backslash \mathcal{X})$ and $p=\frac{2+\alpha}{1+\alpha}$.

Theorem 1.7. Let Assumption A1 hold. Let $u \in \mathcal{X}$ and suppose that there exist $C_{2}=C_{2}(u) \geq 0$ and $\beta \in \mathbb{R}$, such that the Markov semigroup satisfies

$$
\left\|P_{t} u\right\|_{\mathcal{X}}^{2} \leq\|u\|_{\mathcal{X}}^{2}+C_{2} t^{\beta}, \quad \forall t \geq 0
$$

Then

$$
\operatorname{Var}\left(P_{t} u\right) \leq\left(\operatorname{Var}(u)^{\frac{-1}{1+\alpha}}+C_{3} \int_{0}^{t}\left(\|u\|_{\mathcal{X}}^{2}+C_{2} s^{\beta}\right)^{\frac{-1}{1+\alpha}} \mathrm{d} s\right)^{-(1+\alpha)}
$$

where $C_{3}$ is given explicitly (and only depends on $\alpha, C_{1}$ ). In particular, $\operatorname{Var}\left(P_{t} u\right)$ satisfies the following decay rates as $t \rightarrow+\infty$ :

$$
\operatorname{Var}\left(P_{t} u\right) \leq \begin{cases}O\left((\log t)^{-(1+\alpha)}\right) & \beta=1+\alpha \\ O\left(t^{\beta-(1+\alpha)}\right) & 0<\beta<1+\alpha . \\ O\left(t^{-(1+\alpha)}\right) & C_{2}=0 \text { or } \beta \leq 0\end{cases}
$$


Remark 1.8. 1. The choice of space $\mathcal{X}$ is motivated by (1.3): it is beneficial to choose $\mathcal{X}$ that is invariant under the Markov semigroup (i.e., if $u \in \mathcal{X}$ then $P_{t} u \in \mathcal{X}$ for all $\left.t \geq 0\right)$.

2. Clearly, $C_{2}(u)$ is subject to quadratic scaling, for example it can be $C\|u\|_{\mathcal{H}}^{2}$ or $C\|u\|_{\mathcal{X}}^{2}$, but the explicit form is not important.

\subsection{A Generalized Theorem: Departing from the Hilbert Structure}

Theorems 1.6 and 1.7 demonstrate how estimates on the density of states near 0 imply a weak Poincaré inequality and a rate of decay to equilibrium. However, it is not essential to restrict oneself to a subspace $\mathcal{X}$. In fact, it is often desirable to deal with functional spaces that are not contained in $\mathcal{H}$, as it may provide improved estimates and decay rates. In particular, this makes sense when the operator in question is the generator of a Markov semigroup, and acts on a range of spaces simultaneously. Hence, we replace Assumption A1 by a more general one.

Assumption A2. There exist Banach spaces $\mathcal{X}, \mathcal{Y}$ of functions on $M$, a constant $r>0$ and a function $\psi_{\mathcal{X}, \mathcal{Y}} \in L^{1}(0, r)$ that is strictly positive a.e. on $(0, r)$, such that

(1) $\mathcal{X} \cap \mathcal{Y} \cap D(\mathcal{E})$ is dense in $D(\mathcal{E})$ (in the topology of $D(\mathcal{E})$ ).

(2) The mapping $\lambda \mapsto \frac{\mathrm{d}}{\mathrm{d} \lambda}(E(\lambda) u, v)_{\mathcal{H}}$ is continuous on $(0, r)$ for every $u \in$ $\mathcal{X} \cap \mathcal{H}$ and $v \in \mathcal{Y} \cap \mathcal{H}$ and satisfies

$$
\left|\frac{\mathrm{d}}{\mathrm{d} \lambda}(E(\lambda) u, v)_{\mathcal{H}}\right| \leq \psi_{\mathcal{X}, \mathcal{Y}}(\lambda)\|u\|_{\mathcal{X}}\|v\|_{\mathcal{Y}}, \quad \forall \lambda \in(0, r) .
$$

We can now state the following more general theorem.

Theorem 1.9. Let the conditions of Assumption A2 hold, and define $\Psi_{\mathcal{X}, \mathcal{Y}}(\rho)=$ $\int_{0}^{\rho} \psi_{\mathcal{X}, \mathcal{Y}}(\lambda) \mathrm{d} \lambda, \rho \in(0, r)$. Then:

a. There exists $K_{0} \in(0,1)$ such that the following functional inequality holds:

$$
\begin{gathered}
(1-K) \Psi_{\mathcal{X}, \mathcal{Y}}^{-1}\left(K \frac{\operatorname{Var}(u)}{\|u\|_{\mathcal{X}}\|u\|_{\mathcal{Y}}}\right) \operatorname{Var}(u) \leq \mathcal{E}(u), \\
\forall K \in\left(0, K_{0}\right), \forall u \in D(\mathcal{E})
\end{gathered}
$$

where $\|u\|_{\mathcal{X}}=+\infty$ if $u \notin \mathcal{X}$ and similarly for $\mathcal{Y}$.

b. If $\mathcal{X}=\mathcal{Y}$ and $\psi_{\mathcal{X}, \mathcal{Y}}(\lambda)=C_{1} \lambda^{\alpha}, \alpha>-1$, the estimate (1.6) reduces to the $(\Phi, p)-W P I$ as in Definition 1.3 with $\Phi(u)=\|u\|_{\mathcal{X}}^{2}$ and $p=\frac{\alpha+2}{\alpha+1}$.

c. If, in addition, $\mathcal{X}=\mathcal{Y} \subset \mathcal{H}$ then we obtain Theorem 1.6.

Remark 1.10. The inequality (1.6) can be viewed as an implicit form of the weak Poincaré inequality. Note that setting $K=0$ (which is excluded in the theorem) leads to the Poincaré inequality.

The power of this result is demonstrated in the following corollary, where the celebrated Nash inequality is obtained as a simple consequence. This simple derivation is discussed in Remark 4.2. 
Corollary 1.11 (Nash inequality). When $H=-\Delta: H^{2}\left(\mathbb{R}^{d}\right) \subset L^{2}\left(\mathbb{R}^{d}\right) \rightarrow$ $L^{2}\left(\mathbb{R}^{d}\right)$ and $\mathcal{Y}=\mathcal{X}=L^{1}\left(\mathbb{R}^{d}\right)$ the inequality (1.6) is precisely Nash's inequality $[15]$ :

$$
\|u\|_{L^{2}}^{2} \leq C\left(\|\nabla u\|_{L^{2}}^{2}\right)^{\frac{d}{d+2}}\left(\|u\|_{L^{1}}^{2}\right)^{\frac{2}{d+2}}, \quad \forall u \in L^{1}\left(\mathbb{R}^{d}\right) \cap H^{1}\left(\mathbb{R}^{d}\right),
$$

where $C>0$ does not depend on $u$. Furthermore, using Proposition 1.13 an explicit constant may be computed to yield $C=\left(\frac{\left|\mathbb{S}^{d-1}\right|}{2}\right)^{\frac{2}{2+d}} \frac{2+d}{d}$.

Proof. The (simple) proof of this corollary is done by applying our results to the heat semigroup. More details are provided in the examples (Sect. 4), in particular see Remark 4.2.

Remark 1.12. The requirement that $\psi_{\mathcal{X}, \mathcal{Y}}$ is strictly positive a.e. on $(0, r)$, for some $r>0$ (perhaps very small), is quite natural as we are interested in operators that lack a spectral gap. However, one can easily generalize our result even if that is not the case by defining

$$
\Psi_{\mathcal{X}, \mathcal{Y}}^{-1}(y)=\sup \left\{x \in(0, r) \mid \Psi_{\mathcal{X}, \mathcal{Y}}(x) \leq y\right\} .
$$

\subsection{Precise Constants}

Under additional mild assumptions, one can improve Theorem 1.9 by replacing the inequality (1.6) which contains an arbitrary constant $K$ with an inequality that has an explicit constant. The question of how far this constant is from being sharp is the topic of ongoing research.

Proposition 1.13. Let the conditions of Assumption A2 hold. Assume in addition that $\psi_{\mathcal{X}, \mathcal{Y}}$ can be extended to a continuous function on $(0, R)$, where $R \in[r,+\infty]$ is such that if $\Psi_{\mathcal{X}, \mathcal{Y}}(\rho):=\int_{0}^{\rho} \psi_{\mathcal{X}, \mathcal{Y}}(\lambda) \mathrm{d} \lambda, \rho \in(0, R)$ and

$$
g_{\mathcal{X}, \mathcal{Y}}(\rho):=\Psi_{\mathcal{X}, \mathcal{Y}}(\rho)+\rho \psi_{\mathcal{X}, \mathcal{Y}}(\rho)
$$

then $g$ is non-decreasing and $\lim _{\rho \rightarrow 0^{+}} g_{\mathcal{X}, \mathcal{Y}}(\rho)=0, \lim _{\rho \rightarrow R^{-}} g_{\mathcal{X}, \mathcal{Y}}(\rho)=+\infty$. Then:

a. The following functional inequality holds:

$$
\begin{aligned}
& \left(g_{\mathcal{X}, \mathcal{Y}}^{-1}\left(\frac{\operatorname{Var}(u)}{\|u\|_{\mathcal{X}}\|u\|_{\mathcal{Y}}}\right)\right)^{2} \psi_{\mathcal{X}, \mathcal{Y}}\left(g_{\mathcal{X}, \mathcal{Y}}^{-1}\left(\frac{\operatorname{Var}(u)}{\|u\|_{\mathcal{X}}\|u\|_{\mathcal{Y}}}\right)\right)\|u\|_{\mathcal{X}}\|u\|_{\mathcal{Y}} \leq \mathcal{E}(u) \\
& \forall u \in D(\mathcal{E}), \\
& \text { where }\|u\|_{\mathcal{X}}=+\infty \text { if } u \notin \mathcal{X} \text { and similarly for } \mathcal{Y} .
\end{aligned}
$$

b. If $\mathcal{X}=\mathcal{Y}$, and $\psi_{\mathcal{X}, \mathcal{Y}}(\lambda)=C_{1} \lambda^{\alpha}, \alpha>-1$ then the estimate (1.7) reduces to the $(\Phi, p)-W P I$ as in Definition 1.3 with $\Phi(u)=\|u\|_{\mathcal{X}}^{2}, p=\frac{\alpha+2}{\alpha+1}$ and $C=C_{1}^{\frac{1}{2+\alpha}} \frac{2+\alpha}{1+\alpha}$.

Organization of the paper. Before proceeding to prove our theorems, we first discuss both the classical and the weak Poincaré inequalities, and their connection to Markov semigroups in Sect. 2. The proofs will follow in Sect. 3, and we then present various applications of these theorems in Sect. 4, where we shall also prove Corollary 1.11. 


\section{Poincaré Inequalities}

In this section, we recall the famous Poincaré inequality, its connection to Markov semigroups, and we discuss its "weak" variant, the so-called weak Poincaré inequality.

\subsection{The Classical Poincaré Inequality}

When $M$ is a compact Riemannian manifold or a bounded domain of $\mathbb{R}^{d}$, the classical $L^{2}$ Poincaré inequality reads $[3, \S 4.2 .1]$

$$
\int_{M}\left|\varphi(x)-\left(\frac{1}{|M|} \int_{M} \varphi(y) \mathrm{d} y\right)\right|^{2} \mathrm{~d} x \leq C_{M} \int_{M}|\nabla \varphi(x)|^{2} \mathrm{~d} x,
$$

where $|M|$ is the volume of $M$, and $C_{M}>0$ is independent of $u$.

Motivation: the heat semigroup. Let us illustrate why the quantities appearing in this inequality are natural. Let $M \subset \mathbb{R}^{d}$ be a bounded, connected and smooth domain. Consider the heat semigroup, i.e., solutions of

$$
\partial_{t} u(t, x)=\Delta_{x} u(t, x), \quad x \in M, t \in \mathbb{R}_{+},
$$

subject to Neumann boundary conditions with initial data $u(0, x)=u_{0}(x)$. The associated invariant measure is $\mathrm{d} \mu(x)=\frac{\mathrm{d} x}{|M|}$. It is well known that in this case the spectrum of $\Delta_{x}$ is discrete and non-positive. In particular, its kernel is separated from the rest of the spectrum. This immediately implies that $u(t, x)=P_{t} u_{0}(x)$ converges to the projection onto the kernel, given by

$$
P_{\mathrm{ker}} u_{0}:=\int_{M} u_{0}(x) \mathrm{d} \mu(x)
$$

Thus, we are interested in the decay rate as $t \rightarrow+\infty$ of

$$
\mathcal{V}\left(P_{t} u_{0}\right):=\left\|P_{t} u_{0}-P_{\text {ker }}\left(P_{t} u_{0}\right)\right\|_{L^{2}(\mathrm{~d} \mu)}^{2}=\left\|P_{t} u_{0}-P_{\text {ker }} u_{0}\right\|_{L^{2}(\mathrm{~d} \mu)}^{2} .
$$

The entropy method. A common method to obtain decay rates of this type is the so-called entropy method. Given the "relative distance" $\mathcal{V}$ (a Lyapunov functional), we find its production functional $\mathcal{E}$ by formally differentiating along the flow of the semigroup:

$$
\begin{aligned}
\frac{\mathrm{d}}{\mathrm{d} t} \mathcal{V}\left(P_{t} u_{0}\right) & =2\left(\partial_{t} P_{t} u_{0}, P_{t} u_{0}-P_{\mathrm{ker}} u_{0}\right)_{L^{2}(\mathrm{~d} \mu)} \\
& =2 \int_{M} P_{t} u_{0}(x) \Delta_{x} P_{t} u_{0}(x) \mathrm{d} \mu(x)=-2 \mathcal{E}\left(P_{t} u_{0}\right),
\end{aligned}
$$

where $\mathcal{E}$ turns out to be the associated Dirichlet form. Note that since $P_{\text {ker }}=$ $E(\{0\})$ we can rewrite $(2.2)$ as $\frac{\mathrm{d}}{\mathrm{d} t} \operatorname{Var}\left(P_{t} u_{0}\right)=-2 \mathcal{E}\left(P_{t} u_{0}\right)$. Now we seek a pure functional inequality involving $\mathcal{V}$ and $\mathcal{E}$. In particular (see, for example, $[17$, Chapter $3, \S 3.2]$ ), one looks for a functional inequality of the form

$$
\mathcal{E}(u) \geq \Theta(\mathcal{V}(u)), \quad \forall u \in D(\mathcal{E}),
$$


with an appropriate nonnegative function $\Theta$. Succeeding in finding such an inequality entails, in view of (2.2),

$$
\frac{\mathrm{d}}{\mathrm{d} t} \mathcal{V}\left(P_{t} u_{0}\right) \leq-2 \Theta\left(\mathcal{V}\left(P_{t} u_{0}\right)\right)
$$

from which an explicit rate is derived.

Returning to the heat semigroup, we notice that the classical Poincaré inequality (2.1) is exactly a functional inequality of the form of (2.3). Moreover, the linear connection between the variance and the Dirichlet form yields an exponential rate of decay for $\operatorname{Var}\left(P_{t} u_{0}\right)$.

\subsection{Relationship to Markov Semigroups}

In view of Sect. 2.1, there is a natural extension of the notion of a Poincaré inequality to general Markov semigroups. Let $\left\{P_{t}\right\}_{t>0}$ be a Markov semigroup on $\mathcal{H}=L^{2}(M, \mathrm{~d} \mu)$ with a generator $-H$, where $H$ is a self-adjoint, nonnegative operator, and $\mathrm{d} \mu$ its invariant measure. Then the Poincaré inequality, as already defined (Definition 1.1), is

$$
\operatorname{Var}(u) \leq C \mathcal{E}(u), \quad \forall u \in D(\mathcal{E}) .
$$

The following well-known theorem (see [3, Theorem 4.2.5]) serves as a motivation for our current investigation:

Theorem 2.1. The following conditions are equivalent:

(1) $H$ satisfies a Poincaré inequality with constant $C$.

(2) The spectrum of $H$ is contained in $\{0\} \cup\left[\frac{1}{C}, \infty\right)$.

(3) For every $u \in L^{2}(M, \mathrm{~d} \mu)$ and every $t \geq 0$,

$$
\operatorname{Var}\left(P_{t} u\right) \leq e^{-2 t / C} \operatorname{Var}(u)
$$

\subsection{The Weak Poincaré Inequality (WPI)}

It is natural to ask whether one can obtain a generalization of Theorem 2.1 to generators which lack a spectral gap. We note that a differential operator acting on functions defined in an unbounded domain (generically) lacks a spectral gap. Our Theorems 1.6 and 1.9 provide an answer to this question, where the Poincaré inequality is replaced by some form of a weak Poincaré inequality. In the following, we provide a brief review of the existing literature on variants of the weak Poincaré inequality.

This topic has a very rich history, in particular in the second half of the twentieth century. As was hinted in Corollary 1.11, a closely related example is Nash's celebrated inequality [15]:

$$
\|u\|_{L^{2}}^{2} \leq C\left(\|\nabla u\|_{L^{2}}^{2}\right)^{\frac{d}{d+2}}\left(\|u\|_{L^{1}}^{2}\right)^{\frac{2}{d+2}}, \quad \forall u \in L^{1}\left(\mathbb{R}^{d}\right) \cap H^{1}\left(\mathbb{R}^{d}\right)
$$

where $C>0$ does not depend on $u$. Estimates of the same spirit are then developed in [9] for example.

The form of the weak Poincaré inequality which we consider (Definition 1.3) first appeared in [13, Equation (2.3)], where it is also shown how such a differential inequality leads to an algebraic decay rate. These ideas 
were then further developed in $[2,5,16,18-21]$. We also refer to [1] where the notion of a "weak spectral gap" is introduced.

In fact, in the influential work of Röckner and Wang [16] several variants of the WPI were introduced. The most general one is

$$
\operatorname{Var}(u) \leq \alpha(r) \mathcal{E}(u)+r \Phi(u), \quad \forall u \in D(\mathcal{E}), r>0,
$$

where $\alpha:(0, \infty) \rightarrow(0, \infty)$ is decreasing and $\Phi: L^{2}(\mathrm{~d} \mu) \rightarrow[0, \infty]$ satisfies $\Phi(c u)=c^{2} \Phi(u)$ for any $c \in \mathbb{R}$ and $u \in L^{2}(\mathrm{~d} \mu)$. This is equivalent to our $(\Phi, p)$-WPI whenever $\alpha(r)=C r^{1-p}$.

Continuing upon the work of Röckner and Wang and their notion of WPI, works on connections between these inequalities and isoperimetry or concentration properties of the underlying measures have been extremely prolific in the probability community. We refer the interested reader to $[4,6,8,11,12,14]$. For a recent account of the notions discussed here, and in particular the relationship between functional inequalities and Markov semigroups, we refer to the book [3].

\section{Proofs of the Theorems}

We first prove the more general Theorem 1.9 and show how Theorem 1.6 is a straightforward corollary. We then show how to obtain the decay rates in Theorem 1.7, and we conclude with the proof of Proposition 1.13. For brevity, we omit the subscripts from the functions $\psi_{\mathcal{X}, \mathcal{Y}}, \Psi_{\mathcal{X}, \mathcal{Y}}$ and $g_{\mathcal{X}, \mathcal{Y}}$.

\subsection{Proof of Theorem 1.9a}

First, we show that an estimate on the density of states near 0 leads to the WPI (1.6). Let $r_{0} \in(0, r)$ to be chosen later. Let $\{E(\lambda)\}_{\lambda \geq 0}$ be the resolution of the identity of $H$. Let $u \in D(\mathcal{E}) \cap \mathcal{X} \cap \mathcal{Y}$. Then:

$$
\begin{aligned}
\mathcal{E}(u) & =\int_{M} u H u \mathrm{~d} \mu=\int_{M} u \int_{[0, \infty)} \lambda \mathrm{d} E(\lambda) u \mathrm{~d} \mu \\
& \geq \int_{M} u \int_{\left[r_{0}, \infty\right)} \lambda \mathrm{d} E(\lambda) u \mathrm{~d} \mu \geq r_{0} \int_{M} u \int_{\left[r_{0}, \infty\right)} \mathrm{d} E(\lambda) u \mathrm{~d} \mu \\
& =r_{0} \int_{M} u \int_{[0, \infty)} \mathrm{d} E(\lambda) u \mathrm{~d} \mu-r_{0}\|E(\{0\}) u\|_{\mathcal{H}}^{2}-r_{0} \int_{M} u \int_{\left(0, r_{0}\right)} \mathrm{d} E(\lambda) u \mathrm{~d} \mu \\
& =r_{0} \operatorname{Var}(u)-r_{0} \int_{M} u \int_{\left(0, r_{0}\right)} \mathrm{d} E(\lambda) u \mathrm{~d} \mu .
\end{aligned}
$$

We now use the estimate on the density of states (1.5) to obtain

$$
\begin{array}{r}
\int_{M} u \int_{\left(0, r_{0}\right)} \mathrm{d} E(\lambda) u \mathrm{~d} \mu=\int_{\left(0, r_{0}\right)} \frac{\mathrm{d}}{\mathrm{d} \lambda}(E(\lambda) u, u)_{\mathcal{H}} \mathrm{d} \lambda \\
\leq\|u\|_{\mathcal{X}}\|u\|_{\mathcal{Y}} \int_{\left(0, r_{0}\right)} \psi(\lambda) \mathrm{d} \lambda=\|u\|_{\mathcal{X}}\|u\|_{\mathcal{Y}} \Psi\left(r_{0}\right) .
\end{array}
$$

Hence, we have

$$
\mathcal{E}(u) \geq r_{0}\left(\operatorname{Var}(u)-\|u\|_{\mathcal{X}}\|u\|_{\mathcal{Y}} \Psi\left(r_{0}\right)\right)
$$


Let $K \in(0,1)$ and define

$$
r_{0}=\Psi^{-1}\left(K \frac{\operatorname{Var}(u)}{\|u\|_{\mathcal{X}}\|u\|_{\mathcal{Y}}}\right) \quad \text { so that } \quad \Psi\left(r_{0}\right)=K \frac{\operatorname{Var}(u)}{\|u\|_{\mathcal{X}}\|u\|_{\mathcal{Y}}} .
$$

(To satisfy the condition $r_{0}<r$, we may need $K$ to be small.) Then we get

$$
\mathcal{E}(u) \geq r_{0}(1-K) \operatorname{Var}(u)
$$

which completes the proof.

\subsection{Proof of Theorem $1.9 \mathrm{~b}$, c (and Theorem 1.6)}

The proofs follow from the following lemma where we show how (1.6) leads to a $(\Phi, p)$-WPI.

Lemma 3.1. When $\mathcal{X}=\mathcal{Y}$ and $\psi(\lambda)=C_{1} \lambda^{\alpha}, \alpha>-1$, the inequality (1.6) reduces to the $(\Phi, p)-W P I$ with $\Phi(u)=\|u\|_{\mathcal{X}}^{2}$ and $p=\frac{\alpha+2}{\alpha+1}$. Furthermore, if $\mathcal{X}=\mathcal{Y} \subset \mathcal{H}$, we recover Theorem 1.6.

Proof. Let $\psi(\lambda)=C_{1} \lambda^{\alpha}, \alpha>-1$. Then

$$
\Psi(\rho)=C_{1} \int_{0}^{\rho} \lambda^{\alpha} \mathrm{d} \lambda=\frac{C_{1}}{\alpha+1} \rho^{\alpha+1}
$$

so that

$$
\Psi^{-1}(\tau)=\left(\frac{\alpha+1}{C_{1}}\right)^{\frac{1}{\alpha+1}} \tau^{\frac{1}{\alpha+1}}
$$

Hence,

$$
\Psi^{-1}\left(K \frac{\operatorname{Var}(u)}{\|u\|_{\mathcal{X}}^{2}}\right)=\left(\frac{\alpha+1}{C_{1}}\right)^{\frac{1}{\alpha+1}}\left(K \frac{\operatorname{Var}(u)}{\|u\|_{\mathcal{X}}^{2}}\right)^{\frac{1}{\alpha+1}} .
$$

Plugging this into (1.6), we have

$$
\begin{aligned}
\mathcal{E}(u) & \geq(1-K) \Psi^{-1}\left(K \frac{\operatorname{Var}(u)}{\|u\|_{\mathcal{X}}^{2}}\right) \operatorname{Var}(u) \\
& =(1-K)\left(\frac{\alpha+1}{C_{1}}\right)^{\frac{1}{\alpha+1}}\left(K \frac{\operatorname{Var}(u)}{\|u\|_{\mathcal{X}}^{2}}\right)^{\frac{1}{\alpha+1}} \operatorname{Var}(u) \\
& =C^{\prime} \operatorname{Var}(u)^{\frac{\alpha+2}{\alpha+1}}\left(\|u\|_{\mathcal{X}}^{2}\right)^{-\frac{1}{\alpha+1}} .
\end{aligned}
$$

This leads to

$$
\operatorname{Var}(u) \leq C^{\prime \prime} \mathcal{E}(u)^{\frac{\alpha+1}{\alpha+2}}\left(\|u\|_{\mathcal{X}}^{2}\right)^{\frac{1}{\alpha+2}}
$$

which is a $(\Phi, p)$-WPI with $\Phi(u)=\|u\|_{\mathcal{X}}^{2}$ and $p=\frac{\alpha+2}{\alpha+1}$. 


\subsection{Proof of Theorem 1.7}

We show that the growth rate assumption (1.3) leads to a decay of the variance as in (1.4). This proof is rather standard and is included for completeness. Using (2.2), the $(\Phi, p)$-WPI and (1.3), we have:

$$
\begin{aligned}
\frac{\mathrm{d}}{\mathrm{d} t} \operatorname{Var}\left(P_{t} u\right)=-2 \mathcal{E}\left(P_{t} u\right) & \leq-2 C^{\prime} \operatorname{Var}\left(P_{t} u\right)^{\frac{\alpha+2}{\alpha+1}}\left(\left\|P_{t} u\right\|_{\mathcal{X}}^{2}\right)^{-\frac{1}{\alpha+1}} \\
& \leq-2 C^{\prime} \operatorname{Var}\left(P_{t} u\right)^{\frac{\alpha+2}{\alpha+1}}\left(\|u\|_{\mathcal{X}}^{2}+C_{2} t^{\beta}\right)^{-\frac{1}{\alpha+1}}
\end{aligned}
$$

where $C^{\prime}$ is as in the proof of Lemma 3.1. This is an ordinary differential inequality for $y(t):=\operatorname{Var}\left(P_{t} u\right)$ of the form

$$
\dot{y} \leq-A y^{1+a}\left(B+C t^{b}\right)^{-c},
$$

for $a, c, A, B>0, b \in \mathbb{R}$, and $C \geq 0$. We readily obtain

$$
y(t) \leq\left(y(0)^{-a}+a A \int_{0}^{t}\left(B+C s^{b}\right)^{-c} \mathrm{~d} s\right)^{-1 / a}
$$

which yields the bound (1.4). Asymptotically, we have

$$
y(t)=O\left(t^{-1 / a}\right) \text { as } t \rightarrow+\infty, \quad \text { if } C=0 \text { or } b \leq 0 .
$$

Otherwise, it is easy to see that $b c=1$ leads to logarithmic decay, while $b c<1$ leads to polynomial decay. The precise rates are

$$
y(t)= \begin{cases}O\left((\log t)^{-1 / a}\right) \text { as } t \rightarrow+\infty, & b c=1 . \\ O\left(t^{-(1-b c) / a}\right) \text { as } t \rightarrow+\infty, & b c<1 .\end{cases}
$$

This completes the proof of Theorem 1.7.

Remark 3.2 (The constant $C_{3}$ ). It is beneficial to provide a detailed computation of the constant $C_{3}$ appearing in (1.4). The following computations are performed up to a constant $C$ which does not depend on $\alpha, M, \mathcal{H}, \mathcal{X}$ or any other fundamental quantity.

Considering the proof of Theorem 1.7, we see that $C_{3}$ is denoted $a A$ where $a=\frac{1}{\alpha+1}$ and $A=2 C^{\prime}$ with $C^{\prime}=\underbrace{(1-K) K^{\frac{1}{1+\alpha}}}_{\tilde{K}}\left(\frac{\alpha+1}{C_{1}}\right)^{\frac{1}{\alpha+1}}$ where $C_{1}$ and $\alpha$ appear in the bound (1.2). We readily obtain

$$
C_{3}=2 \tilde{K} \frac{1}{\alpha+1}\left(\frac{\alpha+1}{C_{1}}\right)^{\frac{1}{\alpha+1}}=2 \tilde{K}(\alpha+1)^{\frac{-\alpha}{\alpha+1}} C_{1}^{\frac{-1}{\alpha+1}}
$$

In fact, a short computation using the result of Proposition 1.13 yields the even more explicit formula

$$
C_{3}=2(\alpha+2)^{-\frac{\alpha+2}{\alpha+1}}(\alpha+1)^{\frac{1}{\alpha+1}} C_{1}^{\frac{-1}{\alpha+1}} .
$$




\subsection{Proof of Proposition 1.13}

As seen in the Proof of Theorem 1.6 we have that for all $r_{0} \in(0, r)$

$$
\mathcal{E}(u) \geq r_{0}\left(\operatorname{Var}(u)-\|u\|_{\mathcal{X}}\|u\|_{\mathcal{Y}} \Psi\left(r_{0}\right)\right) .
$$

Our goal is to maximize the right-hand side of this inequality. As such, for any $a, b>0$, consider the function

$$
h(\rho)=\rho(a-\Psi(\rho) b) .
$$

By assumption, we can extend $\psi$ to a continuous function on $(0, R)$, so that $h$ is differentiable and we have

$$
h^{\prime}(\rho)=a-g(\rho) b .
$$

As $g$ increases from 0 to $+\infty$, we see that the unique critical point, $\rho=g^{-1}\left(\frac{a}{b}\right)$ is a maximum point of $h$. Thus

$$
\begin{aligned}
\max _{\rho \in(0, R)} h(\rho) & =g^{-1}\left(\frac{a}{b}\right)\left(a-\Psi\left(g^{-1}\left(\frac{a}{b}\right)\right) b\right) \\
& =g^{-1}\left(\frac{a}{b}\right)\left(a-\left[g\left(g^{-1}\left(\frac{a}{b}\right)\right)-g^{-1}\left(\frac{a}{b}\right) \psi\left(g^{-1}\left(\frac{a}{b}\right)\right)\right] b\right) \\
& =g^{-1}\left(\frac{a}{b}\right)^{2} \psi\left(g^{-1}\left(\frac{a}{b}\right)\right) b .
\end{aligned}
$$

Applying this maximization process to the right-hand side of (3.1) with $a=\operatorname{Var}(u)$ and $b=\|u\|_{\mathcal{X}}\|u\|_{\mathcal{Y}}$ yields the desired inequality (1.7).

To show the second part of the theorem, we notice that $\psi(\lambda)$ can be extended to a continuous function on $(0,+\infty)$ with the same formula $C_{1} \lambda^{\alpha}$. The expression for $\Psi$ is $\Psi(\rho)=C_{1} \frac{\rho^{1+\alpha}}{1+\alpha}$. We note that

$$
g(\rho)=C_{1} \frac{2+\alpha}{1+\alpha} \rho^{1+\alpha}
$$

satisfies the conditions $\lim _{\rho \rightarrow 0} g(\rho)=0$ and $\lim _{\rho \rightarrow+\infty} g(\rho)=+\infty$. Since

$$
g^{-1}(y)=\left(\frac{1+\alpha}{C_{1}(2+\alpha)}\right)^{\frac{1}{1+\alpha}} y^{\frac{1}{1+\alpha}}
$$

and

$$
g^{-1}(y)^{2} \psi\left(g^{-1}(y)\right)=C_{1}\left(g^{-1}(y)\right)^{\alpha+2},
$$

we obtain the result by substituting $y=\frac{\operatorname{Var}(u)}{\|u\|_{\mathcal{X}}^{2}}$, thus leading to the inequality

$$
\mathcal{E}(u) \geq C_{1}^{-\frac{1}{1+\alpha}}\left(\frac{1+\alpha}{2+\alpha}\right)^{\frac{2+\alpha}{1+\alpha}}\left(\frac{\operatorname{Var}(u)}{\|u\|_{\mathcal{X}}^{2}}\right)^{\frac{2+\alpha}{1+\alpha}}\|u\|_{\mathcal{X}}^{2} .
$$




\section{Examples}

Here, we consider several notable examples of equations

$$
\begin{cases}\partial_{t} u(t, x)=-H u(t, x), & t \in \mathbb{R}_{+}, x \in \mathbb{R}^{d}, \\ u(0, x)=u_{0}(x), & x \in \mathbb{R}^{d} .\end{cases}
$$

where $H$ is a constant coefficient pseudodifferential operator:

$$
H=P(D) .
$$

With a slight abuse of notation, we write $H=P(\xi)$, where $\xi \in \mathbb{R}^{d}$.

Assumption A3. Assume that there exist $\gamma_{1}>-1$ and $C, \gamma_{2}>0$ so that $P(\xi)$ satisfies the following conditions:

(1) $P(0)=0$,

(2) $C^{-1}|\xi|^{\gamma_{1}+1} \leq P(\xi) \leq C|\xi|^{\gamma_{2}}$, for any $\xi \in \mathbb{R}^{d}$,

(3) $C^{-1}|\xi|^{\gamma_{1}} \leq|\nabla P(\xi)|$, for any $\xi \in \mathbb{R}^{d} \backslash\{0\}$,

(4) $\mathcal{H}^{d-1}\left(\left\{\xi \in \mathbb{R}^{d}: P(\xi)=\lambda\right\}\right) \leq C \lambda^{\frac{d-1}{\gamma_{1}+1}}$, for any $\lambda>0$.

Here, $\mathcal{H}^{d-1}$ is the $d-1$-dimensional Hausdorff measure. (We use the same constant $C$ in all inequalities for simplicity, but one could specify different constants.)

Then since $P(\xi)$ is a multiplication operator, one obtains the following simple expression for the spectral measure $E(\lambda)$ of $H$ :

$$
(E(\lambda) u, v)_{L^{2}}=\int_{P(\xi) \leq \lambda} \widehat{u}(\xi) \overline{\widehat{v}(\xi)} \mathrm{d} \xi .
$$

Let $\mathrm{d} \sigma_{\lambda_{0}}$ denote the uniform Lebesgue measure on the surface $\left\{\xi \in \mathbb{R}^{d}\right.$ : $\left.P(\xi)=\lambda_{0}\right\}$. Then differentiating (4.1) and using the coarea formula, we obtain:

$$
\begin{aligned}
\left|\frac{\mathrm{d}}{\mathrm{d} \lambda}\right|_{\lambda=\lambda_{0}}(E(\lambda) u, v)_{L^{2}\left(\mathbb{R}^{d}\right)} \mid & =\left|\int_{P(\xi)=\lambda_{0}} \frac{1}{|\nabla P(\xi)|} \widehat{u}(\xi) \overline{\widehat{v}(\xi)} \mathrm{d} \sigma_{\lambda_{0}}\right| \\
& \leq\left|\int_{P(\xi)=\lambda_{0}} \frac{1}{|\nabla P(\xi)|} \mathrm{d} \sigma_{\lambda_{0}}\right|\|\widehat{u}\|_{L^{\infty}\left(\mathbb{R}^{d}\right)}\|\widehat{v}\|_{L^{\infty}\left(\mathbb{R}^{d}\right)} \\
& \leq\left|\int_{P(\xi)=\lambda_{0}} \frac{1}{|\nabla P(\xi)|} \mathrm{d} \sigma_{\lambda_{0}}\right|\|u\|_{L^{1}\left(\mathbb{R}^{d}\right)}\|v\|_{L^{1}\left(\mathbb{R}^{d}\right)} \\
& \leq C \int_{P(\xi)=\lambda_{0}}|\xi|-\gamma_{1} \mathrm{~d} \sigma_{\lambda_{0}}\|u\|_{L^{1}\left(\mathbb{R}^{d}\right)}\|v\|_{L^{1}\left(\mathbb{R}^{d}\right)} \\
& \leq C \int_{P(\xi)=\lambda_{0}} \lambda_{0}^{-\gamma_{1} / \gamma_{2}} \mathrm{~d} \sigma_{\lambda_{0}}\|u\|_{L^{1}\left(\mathbb{R}^{d}\right)}\|v\|_{L^{1}\left(\mathbb{R}^{d}\right)} \\
& \leq C \lambda_{0}^{-\frac{\gamma_{1}}{\gamma_{2}}+\frac{d-1}{\gamma_{1}+1}}\|u\|_{L^{1}\left(\mathbb{R}^{d}\right)}\|v\|_{L^{1}\left(\mathbb{R}^{d}\right)} .
\end{aligned}
$$


From Theorem $1.9 \mathrm{~b}$ this bound on the DoS leads to a $(\Phi, p)$-WPI with $\mathcal{X}=$ $L^{1}\left(\mathbb{R}^{d}\right) \cap L^{2}\left(\mathbb{R}^{d}\right), \Phi(u)=\|u\|_{L^{1}}^{2}$ and $p=\frac{2-\frac{\gamma_{1}}{\gamma_{2}}+\frac{d-1}{\gamma_{1}+1}}{1-\frac{\gamma_{1}}{\gamma_{2}}+\frac{d-1}{\gamma_{1}+1}}=1+\frac{\gamma_{2}\left(\gamma_{1}+1\right)}{\gamma_{2} \gamma_{1}-\gamma_{1}\left(\gamma_{1}+1\right)+d \gamma_{2}}$ :

$$
\|u\|_{L^{2}}^{2} \leq C \mathcal{E}(u)^{\frac{\gamma_{2} \gamma_{1}-\gamma_{1}\left(\gamma_{1}+1\right)+d \gamma_{2}}{2 \gamma_{2}\left(\gamma_{1}+\frac{1}{2}\right)-\gamma_{1}\left(\gamma_{1}+1\right)+d \gamma_{2}}}\|u\|_{L^{1}}^{2-\frac{2 \gamma_{2} \gamma_{1}-2 \gamma_{1}\left(\gamma_{1}+1\right)+2 d \gamma_{2}}{2 \gamma_{2}\left(\gamma_{1}+\frac{1}{2}\right)-\gamma_{1}\left(\gamma_{1}+1\right)+d \gamma_{2}}} .
$$

Moreover, if $\gamma_{2}=1+\gamma_{1}$, the power of $\lambda_{0}$ in the bound (4.2) simply becomes $\lambda_{0}^{\frac{d}{\gamma_{2}}-1}$ and then (4.3) simplifies to

$$
\|u\|_{L^{2}}^{2} \leq C \mathcal{E}(u)^{\frac{d}{\gamma_{2}+d}}\|u\|_{L^{1}}^{\frac{2 \gamma_{2}}{\gamma_{2}+d}}
$$

Remark 4.1 (Other functional subspaces). We focus here on solutions lying in $L^{1}$. However, other natural subspaces to consider are the Hilbert subspaces $L^{2, s}\left(\mathbb{R}^{d}\right)$, defined as

$$
L^{2, s}\left(\mathbb{R}^{d}\right):=\left\{u \in L^{2}\left(\mathbb{R}^{d}\right):\|u\|_{L^{2, s}\left(\mathbb{R}^{d}\right)}^{2}:=\int_{\mathbb{R}^{d}}|u(x)|^{2}\left(1+|x|^{2}\right)^{s} \mathrm{~d} x<\infty\right\} .
$$

These are naturally obtained as follows. In the estimate (4.2) above, rather than extract $\widehat{u}$ and $\widehat{v}$ in $L^{\infty}$, one can use the trace lemma to estimate them in $H^{s}$ with $s>1 / 2$ (if the surface is sufficiently regular for the trace lemma to hold). Then, one uses the simple observation that the $L^{2, s}$ norm of a function is the same as the $H^{s}$ norm of its Fourier transform. The main difference is that the power of $\lambda_{0}$ in the resulting inequality will be different.

\subsection{The Laplacian}

For the Laplacian $P(\xi)=|\xi|^{2}$, the associated equation is the heat equation:

$$
\begin{cases}\partial_{t} u(t, x)=\Delta_{x} u(t, x), & t \in \mathbb{R}_{+}, x \in \mathbb{R}^{d}, \\ u(0, x)=u_{0}(x), & x \in \mathbb{R}^{d} .\end{cases}
$$

Assumption A3 is satisfied with $\gamma_{2}=\gamma_{1}+1=2$, so that the DoS is estimated by $\lambda_{0}^{\frac{d}{2}-1}$ :

$$
\left|\frac{\mathrm{d}}{\mathrm{d} \lambda}\right|_{\lambda=\lambda_{0}}(E(\lambda) u, v)_{L^{2}} \mid \leq C \lambda_{0}^{\frac{d}{2}-1}\|u\|_{L^{1}\left(\mathbb{R}^{d}\right)}\|v\|_{L^{1}\left(\mathbb{R}^{d}\right)} .
$$

Then the WPI (4.4) becomes

$$
\|u\|_{L^{2}}^{2} \leq C\|\nabla u\|_{L^{2}}^{\frac{2 d}{2+d}}\|u\|_{L^{1}}^{\frac{4}{2+d}} .
$$

Remark 4.2 (Nash inequality). This functional inequality is precisely the Nash inequality. This demonstrates how our methodology gives a general framework for many known important inequalities, presented in general form in (4.3) and (4.4).

Remark 4.3 (The constant in the Nash inequality). We note that the computation (4.2) can be performed with precise constants in this case. Then, using Proposition 1.13, we may extract a precise constant in (4.5). A simple computation yields the constant $C=\left(\frac{\left|\mathbb{S}^{d-1}\right|}{2}\right)^{\frac{2}{2+d}} \frac{2+d}{d}$. These computations are left to the reader. We note that the optimal constant in the Nash inequality 
has already been obtained long ago by Carlen and Loss [10]. Improving our constant is the subject of ongoing research.

Convergence to equilibrium. We can apply Theorems $1.9 \mathrm{c}$ and 1.7 with $\alpha=$ $\frac{d}{2}-1$ and $\Phi(u)=\|u\|_{L^{1}}^{2}$. Using the fact that the $L^{1}$ norm of solutions to the heat equation does not increase, we have $C_{2}=0$, where $C_{2}$ is the constant appearing in (1.3). The bound (1.4) becomes

$$
\begin{aligned}
\operatorname{Var}(u(t, \cdot)) & \leq\left(\operatorname{Var}\left(u_{0}\right)^{\frac{-1}{1+\alpha}}+C \int_{0}^{t}\left\|u_{0}\right\|_{L^{1}}^{\frac{-2}{1+\alpha}} \mathrm{d} s\right)^{-(1+\alpha)} \\
& =\left(\operatorname{Var}\left(u_{0}\right)^{-\frac{2}{d}}+C\left\|u_{0}\right\|_{L^{1}}^{-\frac{4}{d}} t\right)^{-\frac{d}{2}} \\
& \leq C\left\|u_{0}\right\|_{L^{1}}^{2} t^{-\frac{d}{2}}
\end{aligned}
$$

and we conclude that for every $u_{0} \in L^{1}\left(\mathbb{R}^{d}\right) \cap L^{2}\left(\mathbb{R}^{d}\right)$

$$
\|u(t, \cdot)\|_{L^{2}}^{2}=\operatorname{Var}(u(t, \cdot))=O\left(t^{-\frac{d}{2}}\right), \quad \text { as } t \rightarrow+\infty,
$$

which is the optimal rate for the heat equation. This can be extended to any $u_{0} \in L^{1}\left(\mathbb{R}^{d}\right)$ by density.

\subsection{The Fractional Laplacian}

For $P(\xi)=|\xi|^{2 p}(p \in(0,1))$ Assumption A3 on $P(\xi)$ is satisfied with $\gamma_{2}=$ $\gamma_{1}+1=2 p$, so that the DoS is estimated by $\lambda_{0}^{\frac{d}{2 p}-1}$ and (4.4) becomes

$$
\|u\|_{L^{2}}^{2} \leq C\left\|(-\Delta)^{\frac{p}{2}} u\right\|_{L^{2}}^{\frac{2 d}{2 p+d}}\|u\|_{L^{1}}^{\frac{4 p}{2 p+d}} .
$$

Remark 4.4. There is no reason not to take values of $p$ greater than 1 . However, the restriction to $p \in(0,1)$ is quite common in the literature, and the result below on time decay only applies to $p \in(0,1)$.

Convergence to equilibrium. From [7] we know that $\|u(t, \cdot)\|_{L^{1}} \leq\left\|u_{0}\right\|_{L^{1}}$ and as such, much like the previous example, we conclude that

$$
\operatorname{Var}(u(t, \cdot)) \leq\left(\operatorname{Var}\left(u_{0}\right)^{-\frac{2 p}{d}}+C\left\|u_{0}\right\|_{L^{1}}^{-\frac{4 p}{d}} t\right)^{-\frac{d}{2 p}} \leq C\left\|u_{0}\right\|_{L^{1}}^{2} t^{-\frac{d}{2 p}}
$$

and hence the asymptotic decay rate is

$$
\|u(t, \cdot)\|_{L^{2}}^{2}=\operatorname{Var}(u(t, \cdot))=O\left(t^{-\frac{d}{2 p}}\right), \quad \text { as } t \rightarrow+\infty .
$$

\subsection{Homogeneous Elliptic Operators}

Consider homogenous elliptic operators of the form

$$
P(\xi)=\sum_{|\alpha|=m} a_{\alpha} \xi^{\alpha}, \quad m \in\{2,4,6, \ldots\},
$$

where $\alpha \in \mathbb{N}_{0}^{d}$ is a multi-index with $|\alpha|=\sum_{i=1}^{d} \alpha_{i}$ and where all coefficients $a_{\alpha} \in \mathbb{R}$ are assumed to be such that the operator satisfies Assumption A3. In this case, $m=\gamma_{1}+1=\gamma_{2}$ and the WPI (4.4) becomes

$$
\|u\|_{L^{2}}^{2} \leq C\left\|P^{1 / 2}(D) u\right\|_{L^{2}}^{\frac{2 d}{m+d}}\|u\|_{L^{1}}^{\frac{2 m}{m+d}}
$$


Examples of such operators which are not functions of the Laplacian include:

(1) $P(\xi)=\sum_{i=1}^{d}\left|\xi_{i}\right|^{4}$

(2) $P(\xi)=\sum_{i=1}^{d}\left|\xi_{i}\right|^{2}-\xi_{1} \xi_{2}$.

For these examples, the only nontrivial condition to verify is the condition $\mathcal{H}^{d-1}\left(\left\{\xi \in \mathbb{R}^{d}: P(\xi)=\lambda\right\}\right) \leq C \lambda^{\frac{d-1}{m}}$.

Convergence to equilibrium. In order to prove convergence to an equilibrium state, one has to know how the $L^{1}$ norm behaves under the flow. The authors are not aware of results in the literature for general operators as the ones we consider here. Based on the known results for the Laplacian and the fractional Laplacian, one could ask:

Question 4.5. Is it true that for every homogeneous elliptic operator of order $m$ which satisfies Assumption A3 and which is the generator of a semigroup $\left(P_{t}\right)_{t \geq 0}$ there exist $C_{2}=C_{2}(u) \geq 0$ and $\beta \in \mathbb{R}$ such that for every $t \geq 0$, $\left\|P_{t} u\right\|_{L^{1}}^{2} \leq\|u\|_{L^{1}}^{2}+C_{2} t^{\beta}$ ?

If the answer is "yes," from Theorem 1.7 this conjecture leads to the following rate of convergence to equilibrium:

$$
\operatorname{Var}\left(P_{t} u\right) \leq \begin{cases}O\left((\log t)^{-\frac{d}{m}}\right) & \beta=\frac{d}{m} . \\ O\left(t^{\beta-\frac{d}{m}}\right) & 0<\beta<\frac{d}{m} . \\ O\left(t^{-\frac{d}{m}}\right) & C_{2}=0 \text { or } \beta \leq 0 .\end{cases}
$$

\section{Acknowledgements}

The first author was support by the UK Engineering and Physical Sciences Research Council (EPSRC) Grant EP/N020154/1. The second author was supported by the Austrian Science Fund (FWF) Grant M 2104-N32. The authors thank the referees for their useful comments which helped improve the content and presentation of this work.

Open Access. This article is distributed under the terms of the Creative Commons Attribution 4.0 International License (http://creativecommons.org/licenses/ by/4.0/), which permits unrestricted use, distribution, and reproduction in any medium, provided you give appropriate credit to the original author(s) and the source, provide a link to the Creative Commons license, and indicate if changes were made.

Publisher's Note Springer Nature remains neutral with regard to jurisdictional claims in published maps and institutional affiliations. 


\section{References}

[1] Aida, S.: Uniform positivity improving property, sobolev inequalities, and spectral gaps. J. Funct. Anal. 158, 152-185 (1998)

[2] Aida, S.: Weak Poincaré inequalities on domains defined by Brownian rough paths. Ann. Prob. 32(4), 3116-3137 (2004)

[3] Bakry, D., Gentil, I., Ledoux, M.: Analysis and Geometry of Markov Diffusion Operators. Grundlehren der mathematischen Wissenschaften, vol. 348. Springer, Cham (2014)

[4] Barthe, F., Cattiaux, P., Roberto, C.: Concentration for independent random variables with heavy tails. Appl. Math. Res. eXpress 39-60, 2005 (2005)

[5] Bertini, L., Zegarlinski, B.: Coercive inequalities for Gibbs measures. J. Funct. Anal. 162, 257-286 (1999)

[6] Bobkov, S.G.: Large deviations and isoperimetry over convex probability measures with heavy tails. Electron. J. Probab. 12, 1072-1100 (2007)

[7] Bonforte, M., Sire, Y., Vázquez, J.L.: Optimal existence and uniqueness theory for the fractional heat equation. Nonlinear Anal. Theory Methods Appl. 153, 142-168 (2017)

[8] Cancrini, N., Martinelli, F.: On the spectral gap of Kawasaki dynamics under a mixing condition revisited. J. Math. Phys. 41(3), 1391-1423 (2000)

[9] Carlen, E.A., Kusuoka, S., Stroock, D.W.: Upper bounds for symmetric Markov transition functions. Ann. Inst. Henri Poincar'e Probab. Stat. 23(S2), 245-287 (1987)

[10] Carlen, E.A., Loss, M.: Sharp constant in Nash's inequality. Int. Math. Res. Not. 1993(7), 213-215 (1993)

[11] Cattiaux, P., Gozlan, N., Guillin, A., Roberto, C.: Functional inequalities for heavy tailed distributions and application to isoperimetry. Electron. J. Probab. 15, 346-385 (2010)

[12] Hu, S., Wang, X.: Subexponential decay in kinetic Fokker-Planck equation: weak hypocoercivity. Bernoulli 25(1), 174-188 (2019)

[13] Liggett, T.M.: $L_{2}$ rates of convergence for attractive reversible nearest particle systems: the critical case. Ann. Probab. 19(3), 935-959 (1991)

[14] Mourrat, J.C.: Variance decay for functionals of the environment viewed by the particle. Ann. l'inst. Henri Poincare (B) Probab. Stat. 47(1), 294-327 (2011)

[15] Nash, J.F.F.: Continuity of solutions of parabolic and elliptic equations. Am. J. Math. 80(4), 931 (1958)

[16] Röckner, M., Wang, F.-Y.: Weak Poincare inequalities and L2-convergence rates of markov semigroups. J. Funct. Anal. 185, 564-603 (2001)

[17] Villani, C.: A review of mathematical topics in collisional kinetic theory. In: Friedlander, S., Serre, D. (eds.) Handbook of Mathematical Fluid Dynamics, vol. 1. Elsevier, Amsterdam (2002)

[18] Wang, F.-Y.: Functional inequalities and spectrum estimates: the infinite measure case. J. Funct. Anal. 194, 288-310 (2002)

[19] Wang, F.-Y.: Functional inequalities on abstract Hilbert spaces and applications. Math. Z. 246(1-2), 359-371 (2004)

[20] Wang, F.-Y.: Weak Poincaré inequalities on path spaces. Int. Math. Res. Not. 2004(2), 89-108 (2004) 
[21] Wang, F.-Y.: Super and weak Poincaré inequalities for hypoelliptic operators. Acta Math. Appl. Sin. Engl. Ser. 25(4), 617-630 (2009)

Jonathan Ben-Artzi

School of Mathematics

Cardiff University

Cardiff CF24 4AG Wales

UK

e-mail: Ben-ArtziJ@cardiff.ac.uk

\section{Amit Einav}

Institut für Mathematik und Wissenschaftliches Rechnen

Karl-Franzens-Universität Graz

Graz

Austria

e-mail: amit.einav@uni-graz.at

Communicated by Jan Derezinski.

Received: January 11, 2019.

Accepted: October 9, 2019. 